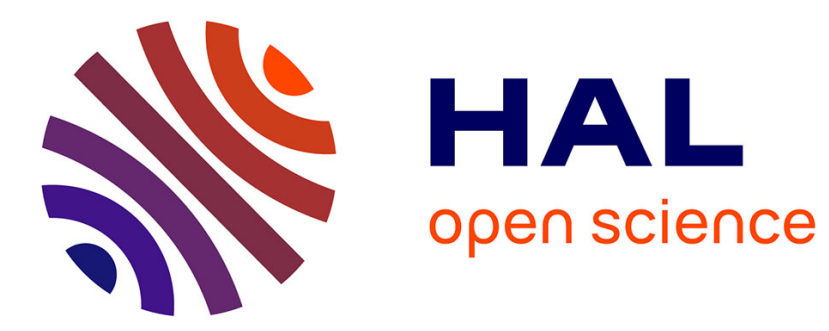

\title{
Steinberg-like theorems for backbone colouring
} Julio Araujo, Frédéric Havet, Mathieu Schmitt

\section{To cite this version:}

Julio Araujo, Frédéric Havet, Mathieu Schmitt. Steinberg-like theorems for backbone colouring. Discrete Applied Mathematics, 2018, 10.1016/j.dam.2017.03.009 . hal-01796713

\section{HAL Id: hal-01796713 \\ https://hal.inria.fr/hal-01796713}

Submitted on 21 May 2018

HAL is a multi-disciplinary open access archive for the deposit and dissemination of scientific research documents, whether they are published or not. The documents may come from teaching and research institutions in France or abroad, or from public or private research centers.
L'archive ouverte pluridisciplinaire HAL, est destinée au dépôt et à la diffusion de documents scientifiques de niveau recherche, publiés ou non, émanant des établissements d'enseignement et de recherche français ou étrangers, des laboratoires publics ou privés. 


\title{
Steinberg-like theorems for backbone colouring
}

\author{
J. Araujo \\ F. Havet \\ M. Schmitt
}

Draft of November 21, 2016

\begin{abstract}
A function $f: V(G) \rightarrow\{1, \ldots, k\}$ is a (proper) $k$-colouring of $G$ if $|f(u)-f(v)| \geq 1$, for every edge $u v \in E(G)$. The chromatic number $\chi(G)$ is the smallest integer $k$ for which there exists a proper $k$-colouring of $G$.

Given a graph $G$ and a subgraph $H$ of $G$, a circular $q$-backbone $k$-colouring $f$ of $(G, H)$ is a $k$ colouring of $G$ such that $q \leq|c(u)-c(v)| \leq k-q$, for each edge $u v \in E(H)$. The circular $q$-backbone chromatic number of a graph pair $(G, H)$, denoted $\operatorname{CBC}_{q}(G, H)$, is the minimum $k$ such that $(G, H)$ admits a circular $q$-backbone $k$-colouring.

Steinberg conjectured that if $G$ is planar and $G$ contains no cycles on 4 or 5 vertices, then $\chi(G) \leq 3$. If this conjecture is correct, then one could deduce that $\mathrm{CBC}_{2}(G, H) \leq 6$, for any $H \subseteq G$. In this work, we first show that if $G$ is a planar graph containing no cycle on 4 or 5 vertices and $H \subseteq G$ is a forest, then $\mathrm{CBC}_{2}(G, H) \leq 7$. Then, we prove that if $H \subseteq G$ is a forest whose connected components are paths, then $\mathrm{CBC}_{2}(G, H) \leq 6$.
\end{abstract}

\section{Introduction}

In this paper, all graphs are considered to be simple and we use standard terminology as the one in [2].

Let $G=(V, E)$ be a graph, and $H=(V, E(H))$ be a spanning subgraph of $G$, called its backbone. A $k$-colouring of $G$ is a mapping $f: V \rightarrow\{1, \ldots, k\}$. Let $f$ be a $k$-colouring of $G$. It is a proper colouring if $|f(u)-f(v)| \geq 1$ for every edge $u v \in E(G)$.. It is a $q$-backbone colouring for $(G, H)$ if $f$ is a proper colouring of $G$ and $|f(u)-f(v)| \geq q$ for all edges $u v \in E(H)$. The chromatic number $\chi(G)$ is the smallest integer $k$ for which there exists a proper $k$-colouring of $G$. The q-backbone chromatic number of $(G, H)$, denoted by $\mathrm{BBC}_{q}(G, H)$, is the smallest integer $k$ for which there exists a $q$-backbone $k$-colouring of $(G, H)[3]$.

Note that if $f$ is a proper $k$-colouring of $G$, then the function $g: V \rightarrow\{1, \ldots, q \cdot k-q+1\}$ defined by $g(v)=q \cdot f(v)-q+1$ is a $q$-backbone colouring of $(G, H)$, for any spanning subgraph $H$ of $G$. Moreover it is well-known that if $G=H$ and $f$ is a proper $\chi(G)$-colouring of $G$, this $q$-backbone colouring $g$ of $(G, H)$ is optimal. Therefore, since $\mathrm{BBC}_{q}(H, H) \leq \mathrm{BBC}_{q}(G, H) \leq \mathrm{BBC}_{q}(G, G)$, we have

$$
q \cdot \chi(H)-q+1 \leq \mathrm{BBC}_{q}(G, H) \leq q \cdot \chi(G)-q+1 .
$$

One can generalise the notion of backbone colouring by allowing a more complicated structure of the colour space. A natural choice is to impose a circular metric on the colours. We can see $\mathbb{Z}_{k}{ }^{1}$ as a cycle of length $k$ with vertex set $\{1, \ldots, k\}$ together with the graphical distance $|\cdot|_{k}$. Then $|a-b|_{k} \geq q$ if and only if $q \leq|a-b| \leq k-q$. A circular $q$-backbone $k$-colouring of $G$ or $q$-backbone $\mathbb{Z}_{k}$-colouring of $(G, H)$ is a mapping $f: V(G) \rightarrow \mathbb{Z}_{k}$ such that $c(v) \neq c(u)$, for each edge $u v \in E(G)$, and $|c(u)-c(v)|_{k} \geq q$ for each edge $u v \in E(H)$. The circular q-backbone chromatic number of a graph pair $(G, H)$, denoted $\mathrm{CBC}_{q}(G, H)$, is the minimum $k$ such that $(G, H)$ admits a circular $q$-backbone $k$-colouring.

Note that if $f$ is a circular $q$-backbone $k$-colouring of $(G, H)$, then $f$ is also a $q$-backbone $k$-colouring of $(G, H)$. On the other hand, observe that a $q$-backbone $k$-colouring $f$ of $(G, H)$ is a circular $q$-backbone $(k+q-1)$-colouring of $(G, H)$. Hence for every graph pair $(G, H)$, where $H$ is a spanning subgraph of $G$, we have

$$
\mathrm{BBC}_{q}(G, H) \leq \mathrm{CBC}_{q}(G, H) \leq \mathrm{BBC}_{q}(G, H)+q-1
$$

\footnotetext{
${ }^{1}$ Whenever we refer to $\mathbb{Z}_{k}$, we mean the group of integers modulo $k$, also denoted by $\mathbb{Z} / n \mathbb{Z}$.
} 
Combining Inequalities (1) and (2), we observe that

$$
q \cdot \chi(H) \leq \mathrm{CBC}_{q}(G, H) \leq q \cdot \chi(G) .
$$

In this paper, we focus on the particular case when $G$ is a planar graph and $H$ is a forest (i.e. an acyclic graph). Inequality (1) and the Four-Colour Theorem [1] imply that for any planar graph $G$ and spanning subgraph $H, \mathrm{BBC}_{q}(G, H) \leq 3 q+1$. However, for $q=2$, Broersma et al. [3] conjectured that this is not best possible if the backbone is a forest.

Conjecture 1. If $G$ is planar and $H$ is a forest in $G$, then $\mathrm{BBC}_{2}(G, H) \leq 6$.

This conjecture would be tight even if $H$ is a Hamilton path as there are examples of planar graph $G$ and Hamilton path $H$ in $G$, for which $\mathrm{BBC}_{2}(G, H)=6$. Furthermore, Havet et al. [6] proved that given a planar graph $G$ and a Hamilton path $H$ in $G$, it is NP-complete to decide whether $\mathrm{BBC}_{2}(G, H) \leq 5$. Campos et al. [4] proved Conjecture 1 when $H$ is a tree of diameter at most 4.

For larger values of $q$, Havet et al. [6] proved the following.

Theorem 2. If $G$ is planar and $H$ is a forest in $G$, then $\operatorname{BBC}_{q}(G, H) \leq q+6$.

For $q \geq 4$, they also show that Theorem 2 is best possible. On the other hand, they conjecture that if $q=3$, Theorem 2 is not best possible.

Conjecture 3. If $G$ is planar and $H$ is a forest in $G$, then $\mathrm{BBC}_{3}(G, H) \leq 8$.

Regarding circular backbone colouring, Havet et al. [6] proved the following.

Theorem 4. If $G$ is planar and $H$ is a forest in $G$, then $\mathrm{CBC}_{q}(G, H) \leq 2 q+4$.

They also conjectured that this upper bound can be reduced by at least 1 .

Conjecture 5. If $G$ is planar and $H$ is a forest in $G$, then $\mathrm{CBC}_{q}(G, H) \leq 2 q+3$.

Observe that Inequalities (1) and (3) imply that Conjectures 1, 3, and 5 for $q \leq 3$, hold if $G$ is has a 3-colouring. There are many sufficient conditions implying that a planar graph has a 3-colouring. For example, the celebrated Grötzsch's Theorem [5] asserts that planar graphs with girth at least 4 admit a 3 -colouring.

One of the most famous conjectures on planar graphs was posed in 1976 by Steinberg (see [7]).

Conjecture 6 (Steinberg, 1976). If $G$ is a planar graph with no cycles of length 4 or 5 , then $\chi(G) \leq 3$.

Note that this long standing conjecture could be proved by showing that $\mathrm{CBC}_{2}(G, G)=6$, for a planar graph $G$ containing no $C_{4}$ nor $C_{5}$ as a subgraph.

In this paper, we give evidences to the above conjectures. We first settle Conjecture 5 for $q=2$ when $G$ is a planar graph without cycles of length 4 or 5 .

Theorem 7. If $G$ is a planar graph with no cycles of length 4 or 5 and $H$ is a forest in $G$, then $\mathrm{CBC}_{2}(G, H) \leq 7$.

We then improve the upper bound when $H$ is a path forest. A forest is a path forest if all its connected components are paths.

Theorem 8. If $G$ is a planar graph without cycles of length 4 or 5 , and $H$ is a path forest in $G$, then $C B C_{2}(G, H) \leq 6$.

Hence when $G$ is a planar graph with no cycles of length 4 or 5 and $H$ is a path forest, Conjecture 1 and Conjecture 5 for $q=2$ hold. It also implies that Conjecture 5 for $q=3$ holds for such graph pair $(G, H)$ thanks to the following lemma.

Lemma 9. Let $k$ and $q$ be positive integers, $G$ a graph and $H$ a subgraph of $G$. If $C B C_{q}(G, H) \leq q \times k$, then $C B C_{q^{\prime}}(G, H) \leq q^{\prime} \times k$, for all $q^{\prime} \geq q$. 
Proof. Let $c$ be a $q$-backbone $q k$-colouring of $(G, H)$. Define $k$ intervals of colours $I_{j}=\{(j-1) q+$ $1, \ldots, j q\}$, for every $1 \leq j \leq k$. We now define a proper $q^{\prime}$-backbone $q^{\prime} k$-colouring of $(G, H) c^{\prime}$ as the following: if $c(v) \in I_{j}$, then $c^{\prime}(v)=c(v)+(j-1)\left(q^{\prime}-q\right)$, for every $1 \leq j \leq k$ and $v \in V(G)$. Note that $c^{\prime}$ must be a proper colouring of $G$ since $c$ is a proper colouring of $G$. Moreover, for an edge $u v \in E(H)$, we have that $c(u)$ and $c(v)$ cannot lie on the same interval $I_{j}$, for some $1 \leq j \leq k$. Thus, $k-q^{\prime}=(k-q)+\left(q^{\prime}-q\right) \geq|c(u)-c(v)| \geq q+\left(q^{\prime}-q\right)=q^{\prime}$.

Corollary 10. If $G$ is a planar graph without cycles of length 4 or 5 , and $H$ is a path forest in $G$, then $C B C_{3}(G, H) \leq 9$.

The remainder of this paper is devoted to the proofs of Theorems 7 and 8 . Both results are proved by supposing that a minimal counter-example with respect to the number of vertices exists. In Section 2 , we present general properties of such counter-examples that we use in both proofs. Then, we prove Theorems 7 and 8 in Sections 3 and 4, respectively.

\section{Preliminaries}

In this section, we first introduce some useful definitions and notations. We then establish properties that we shall use to prove the two above-mentioned theorems. We first prove simple properties of a planar graph without cycles on 4 or 5 vertices, then we establish some properties of minimal counter-examples to Theorems 7 and 8 .

\subsection{Definitions and notations}

Let $S \subseteq V(G)$ be a subset of vertices of $G$ and $F \subseteq E(G)$ be a subset its edges. We denote by $G[S]$ the subgraph of $G$ induced by $S$, by $G \backslash F$ the graph obtained from $G$ by removing the edges in $F$ from its edge set $E(G)$, by $G-S$ the graph $G[V(G) \backslash S]$, by $(G, H)-S$ the graph pair $(G-S, H-S)$, by $(G, H)[S]$ the graph pair $(G[S], H[S])$ and by $(G, H) \backslash F$ the graph pair $((G \backslash F),(H \backslash F))$.

Let $(G, H)$ and $\left(G^{\prime}, H^{\prime}\right)$ be graph pairs such that $H \subseteq G$ and $H^{\prime} \subseteq G^{\prime}$. We say that $\left(G^{\prime}, H^{\prime}\right)$ is a subpair of $(G, H)$ if $H^{\prime} \subseteq H$ and $G^{\prime} \subseteq G$. We say that $\left(\bar{G}^{\prime}, H^{\prime}\right)$ is a proper subpair of $(G, H)$ if it is a subpair of $(G, H)$ and $H^{\prime} \subset H$ or $G^{\prime} \subset G$. Note that the previous or condition is not exclusive.

A graph pair $(G, H)$ is $(k, 2)$-minimal if $C B C_{2}(G, H)>k$, but $C B C_{2}\left(G^{\prime}, H^{\prime}\right) \leq k$ for every proper subpair $\left(G^{\prime}, H^{\prime}\right)$ of $(G, H)$.

For every colour $1 \leq a \leq k$, let $[a]_{k}$ be the set of colours $b \in\{1, \ldots, k\}$ satisfying $|a-b|<2$ or $|a-b|>k-2$. Note that $[a]_{k}$ has always 3 colours.

We say that $c$ is a partial circular 2-backbone $k$-colouring of $(G, H)$ if $c$ is a circular 2-backbone $k$-colouring of a subpair of $(G, H)$. Let $c$ be a partial circular 2-backbone $k$-colouring of a graph pair $(G, H)$. We say that a colour $a$ is available (or possible) at vertex $v \in V(G)$ if none of its neighbours in $G$ is coloured $a$ and none of its neighbours in $H$ has a colour in $[a]_{k}$. We denote by $\operatorname{Av}_{c}(G, H, v)$ the set of available colours at $v$ in $c$.

We emphasize that this definition of available colour does not require that the vertex $v$ is not coloured. If $v$ is already coloured, observe that it can be recoloured by any available colour and we obtain another feasible partial circular 2-backbone colouring of the same subpair of $(G, H)$.

Similarly, we say that a colour $a$ is forbidden at vertex $v$ due to $S \subseteq N_{G}(v)$ if there exists $u \in S \cap N_{G}(v)$ coloured $a$, or if there exists $u \in S \cap N_{H}(v)$ with colour in $[a]_{k}$. When we consider $S=N_{G}(v)$, we simply mention forbidden at vertex $v$. We denote the set of forbidden colours at $v$ in $c$ by $\mathrm{Fb}_{c}(G, H, v)$.

We also define a colour $a$ is strongly forbidden at vertex $v$ due to $S \subseteq N_{G}(v)$ if one cannot obtain a partial circular 2-backbone $k$-colouring by assigning to $v$ the colour $a$ and (re)colouring each vertex $u \in S$ with one colour in $\operatorname{Av}_{c}(G, H, u)$.

We often omit $G, H$ and $c$ from these notations when they are clear in the context. Observe that $\operatorname{Av}(v)=\mathbb{Z}_{k} \backslash \mathrm{Fb}(v)$.

In the following sections, the graph $H$ is a forest. Then, a vertex of degree 0 (resp. 1, at least 2) in $H$ is called an isolated vertex (resp. a leaf, a node). We also denote by leaf $(H)$ the number of leaves in $H$, by isol $(H)$ the number of isolated vertices in $H$ and by $\operatorname{comp}(H)$ the number of its connected components. 


\section{Planar graphs with no cycles of length 4 or 5 .}

A well-known result on planar graphs is the Euler's Formula:

Theorem 11 (Euler's Formula). If $G$ is a connected plane graph, then

$$
|V(G)|-|E(G)|+|F(G)|=2 .
$$

Let $\tau(G)$ denote the number of triangles of a graph $G$.

Lemma 12. If $G$ is a planar graph with no $C_{4}$, then the three statements hold:

(i) every two (not identical) triangles do not share an edge;

(ii) every vertex $v \in V(G)$ belongs to at most $\left\lfloor d_{G}(v) / 2\right\rfloor$ triangles;

(iii) $3 \tau(G) \leq|E(G)|$.

Proof. To prove (i), observe that two triangles with different vertex sets cannot share at least one edge, otherwise there would be a $C_{4}$ in $G$. (ii) follows directly from (i) by observing that the graph induced by the neighbourhood of $v$ contains no path of length 2, since there is no 4-cycles. Finally, we derive (iii) from (ii). Indeed (ii) implies $3 \tau(G) \leq \sum_{v \in V(G)}\left\lfloor d_{G}(v) / 2\right\rfloor$. Since $\sum_{v \in V(G)} d_{G}(v) / 2=|E(G)|$, we obtain $3 \tau(G) \leq|E(G)|$.

Lemma 13. If $G$ is a connected planar graph with no cycles of length 4 or 5 and $G \neq K_{3}$, then $|E(G)| \leq 2|V(G)|-4$.

Proof. Without loss of generality, we may assume tat $G$ is embedded in the plane. By Euler's Formula, we have $6|E(G)|=6|V(G)|+6|F(G)|-12$. Hence

$$
4|E(G)|=6|V(G)|-\sum_{f \in F(G)}(d(f)-6)-12 \leq 6|V(G)|+3\left|F_{3}(G)\right|-12
$$

where $F_{3}(G)$ is the set of 3 -faces in $G$. Since $G \neq K_{3}$, we observe that $\left|F_{3}(G)\right| \leq \tau(G)$, and then $3\left|F_{3}(G)\right| \leq|E(G)|$, by Lemma 12 -(iii). Hence, $3|E(G)| \leq 6|V(G)|-12$, that is $|E(G)| \leq 2|V(G)|-4$.

\section{Properties of $(k, 2)$-minimal pairs}

Lemma 14. Let $(G, H)$ be a $(k, 2)$-minimal pair and c be a partial 2-backbone $k$-colouring of $(G, H)$. If $u v \in E(H)$ and $1 \leq|\operatorname{Av}(u)| \leq 3$, then at most $4-|\operatorname{Av}(u)|$ colours are strongly forbidden at $v$ due to $u$.

Proof. Observe that a colour cannot be assigned to $v$ due to $u$ with $u v \in E(H)$ if and only if it is in the set $F=\bigcap_{a \in \operatorname{Av}(u)}[a]_{k}$. Note that this set $F$ is maximized when $\operatorname{Av}(u)$ has consecutive colours. Thus, $F$ has at most 3 colours when $|\operatorname{Av}(u)|=1, F$ has at most 2 colours when $|\operatorname{Av}(u)|=2$ and $F$ has at most 1 colour when $|\operatorname{Av}(u)|=3$.

The total degree of a vertex $v$ in $(G, H)$ is $d^{t}(v, G, H)=d_{G}(v)+2 d_{H}(v)$. We often simplify the notation $d^{t}(v, G, H)$ to $d^{t}(v)$ when $G$ and $H$ are clear from the context. Note that $d^{t}(v)$ is an upper bound on the maximum number of forbidden colours at $v$, when extending a circular 2-backbone $k$-colouring of $(G, H)-v$ to $v$.

Lemma 15. If $(G, H)$ is a $(k, 2)$-minimal pair, then $G$ is connected.

Proof. By contradiction, suppose that $G$ is not connected and let $C \subset V(G)$ be a connected component of $G$. Since $(G, H)$ is $(k, 2)$-minimal, observe that $(G, H)-C$ and $(G, H)[C]$ admit circular 2-backbone $k$-colourings $c$ and $c^{\prime}$, respectively. Combining $c$ and $c^{\prime}$, one obtains a circular 2-backbone $k$-colouring of $(G, H)$. This is a contradiction to the hypothesis that $C B C_{2}(G, H)>k$.

Lemma 16. If $(G, H)$ is a $(k, 2)$-minimal pair, then $d^{t}(v) \geq k$, for all $v \in V(G)$.

Proof. Assume for a contradiction that there is a vertex $v$ such that $d^{t}(v)<k$. By minimality of $(G, H)$, $(G, H)-v$ admits a circular 2-backbone $k$-colouring $c$. Now at most $d^{t}(v)$ colours are forbidden at $v$. Hence $c$ can be extended into a circular 2-backbone $k$-colouring of $(G, H)$, a contradiction. 
Lemma 17. Let $(G, H)$ be a $(k, 2)$-minimal pair. If $u v \in E(H)$, then $d^{t}(u)+d^{t}(v) \geq 2 k+2$.

Proof. Let $x \in\{u, v\}$. Set $r(x)=d^{t}(x)-k$. If $r(x) \geq 2$, then we have the result by Lemma 16 . So we may assume that $r(x) \leq 1$. By minimality of $(G, H)$, there exists a circular 2-backbone $k$-colouring $c$ of $(G, H)-\{u, v\}$. Since $u v \in E(H)$, the total degree of $x$ in $(G, H)-\{u, v\}$ is $k+r(x)-3$. Hence there is a set $\operatorname{Av}(x)$ of $k-(k+r(x)-3)=3-r(x)$ available colours at $x$.

Let $F$ be the set of strongly forbidden colors at $u$ due to $v$. By Lemma $14,|F| \leq r(u)+1$. Since $(G, H)$ is a $(k, 2)$-minimal pair, the colouring $c$ cannot be modified into a circular 2-backbone $k$-colouring of $(G, H)$. Thus $|F| \geq|\operatorname{Av}(x)|$, so $r(u)+1 \geq 3-r(v)$. Hence $d^{t}(u)+d^{t}(v) \geq 2 k+2$.

\section{Forest backbone}

The aim of this section is to prove the following.

Theorem 7. If $G$ is a planar graph with no cycles of length 4 or 5 and $H$ is a forest in $G$, then $\mathrm{CBC}_{2}(G, H) \leq 7$.

From this point to the end of Section 3, let $(G, H)$ be a minimal counter-example to Theorem 8 and whenever we use the notation $[a]$, when $a$ is a colour, we mean $[a]_{7}$. By Lemmas 15,16 and 17 , observe that $G$ must be connected, each vertex must have total degree at least 7 and, for each edge $u v \in H$, $d^{t}(u)+d^{t}(v) \geq 16$. We now prove extra properties of such a counter-example.

Lemma 18. Let $P=u v w$ be a path in $H$ and $c$ be a circular 2-backbone 7-colouring of $(G, H)-\{u, v, w\}$. If $|\operatorname{Av}(u)|=3$ and $|\operatorname{Av}(w)|=3$, then at most 3 colours are strongly forbidden at $v$ due to $\{u, w\}$ (no matter whether $u w \in E(G))$.

Proof. Suppose $|\operatorname{Av}(u)|=3$ and $|\operatorname{Av}(w)|=3$. Note that if we show that at most 3 colours are strongly forbidden when $u w \in E(G)$, then we also show that the same holds when $u w \notin E(G)$. In fact, we have the same amount of colours and one constraint less, the one that $u$ and $w$ must receive disjoint colours, to extend $c$ to $(G, H)$. So let us assume $u w \in E(G)$.

Let $A_{1}=\operatorname{Av}(u)$ and $A_{2}=a v(w)$. Let $N$ be the set colours of $\mathbb{Z}_{7}$ that are consecutive to some colour of $\operatorname{Av}(u)$. Observe that $|N| \geq 4$ and that $|N|=4$ if and only if $\operatorname{Av}(u)=\{i, i+2, i+4\}$ for some $i$ and $N=\mathbb{Z}_{7} \backslash \operatorname{Av}(u)$.

If $N \cap \operatorname{Av}(w)=\emptyset$, then $|N|=\left|\mathbb{Z}_{7}\right|-|\operatorname{Av}(w)|=4$. It follows that $\operatorname{Av}(w)=\operatorname{Av}(u)=\{i, i+2, i+4\}$ for some $i$, and the set of strongly forbidden colours at $v$ is $\{i+1, i+3\}$ (recall that $u w \in E(G)$ ).

Henceforth we may assume that $N$ and $\operatorname{Av}(w)$ intersect. Thus there are two consecutive colours $a_{1} \in \operatorname{Av}(u)$ and $a_{2} \in \operatorname{Av}(w)$. Without loss of generality, we may assume that $a_{2}=a_{1}+1$. Observe that the three colours not in $\left[a_{1}\right] \cup\left[a_{2}\right]$ are not strongly forbidden at $v$.

If $a_{1}-1$ or $a_{2}+1$ is not strongly forbidden, we have the result. So we may assume that both $a_{1}-1$ and $a_{2}+1$ are strongly forbidden. Then $\operatorname{Av}(u)=\left[a_{1}-1\right]$ or $\left[a_{1}-1\right]$ contains exactly two colours of each set $\operatorname{Av}(u)$ and $\operatorname{Av}(w)$ and the colour of $\operatorname{Av}(u) \backslash\left[a_{1}-1\right]$ is the same as the color of $\operatorname{Av}(w) \backslash\left[a_{1}-1\right]$. Similarly, either $\operatorname{Av}(w)=\left[a_{2}+1\right]$ or $\left[a_{2}+1\right]$ contains exactly two colours of $\operatorname{Av}(u)$ and exactly two colours of $\operatorname{Av}(w)$ and the colour of $\operatorname{Av}(u) \backslash\left[a_{2}+1\right]$ is the same as the colour of $\operatorname{Av}(w) \backslash\left[a_{2}+1\right]$. Consequently, we necessarily have $\operatorname{Av}(u)=\left[a_{1}-1\right]$ and $\operatorname{Av}(w)=\left[a_{2}+1\right]$. Now $a_{1}$ and $a_{2}$ are not strongly forbidden at $v$ and there are just two strongly forbidden colours at $v$ due to $\{u, w\}$.

Lemma 19. If $S=\left\{s_{1}, \ldots, s_{t}\right\}$ is the set of neighbours of $v$ in $H$ such that $d^{t}\left(s_{i}\right)=7$, for every $1 \leq i \leq t$, then $d^{t}(v) \geq 8+t$.

Proof. The total degree of $v$ in $(G, H)$ is $d^{t}(v)=3 t+\tilde{d}$ with $\tilde{d}$ the total degree of $v$ in $(G, H)-S$. In particular, if $t \geq 4$, then $d^{t}(v) \geq 3 t \geq 8+t$. Therefore, we may assume that $t \leq 3$. If $t=1$, then Lemma 17 yields the results. Henceforth, we now assume $t \in\{2,3\}$.

By minimality of $(G, H)$, there exists a circular 2-backbone 7-colouring $c$ of $(G, H)-(S \cup\{v\})$. In $(G, H)-(S \cup\{v\})$, the total degree of $s_{i}$ is at most 4 . Hence, there is a set $A_{i}$ of at least 3 available colours at $s_{i}$.

Let $F_{1,2}$ (resp. $F_{3}$ ) be the set of strongly forbidden colours at $v$ by $\left\{s_{1}, s_{2}\right\}$ (resp. $\left\{s_{3}\right\}$ ). By Lemmas 17 and 18, we have $\left|F_{3}\right| \leq 4-\left|A_{3}\right| \leq 1$ and $\left|F_{1,2}\right| \leq 3$.

Since $(G, H)$ is minimal, then all seven colours must be strongly forbidden at $v$. If $t=2$, this yields $7 \leq \tilde{d}+\left|F_{1,2}\right|$. But $d^{t}(v)=\tilde{d}+6$. So $d^{t}(v) \geq 13-\left|F_{1,2}\right| \geq 10$. 
If $t=3$, this yields $7 \leq \tilde{d}+\left|F_{1,2}\right|+\left|F_{3}\right|$. But $d^{t}(v)=\tilde{d}+9$. So $d^{t}(v) \geq 16-\left|F_{1,2}\right|-\left|F_{3}\right| \geq 11$.

Corollary 20. If $(G, H)$ be a $(7,2)$-minimal pair such that $G$ is a planar graph and $H$ is a spanning forest of $G$, then

$$
\sum_{v \in V(G)} d^{t}(v) \geq 8|V(G)|-\operatorname{isol}(H)
$$

Proof. Recall that Lemma 16 states that $d^{t}(v) \geq 7$, for every $v \in V(G)$. In particular, if $v$ is an isolated vertex of $H$, then $d^{t}(v) \geq 7$. If $v$ is not isolated and $d^{t}(v)=7$, then $v$ each neighbour $u$ of $v$ in $H$ has total degree at least 9 , by Lemma 17 .

Let $u$ be a vertex such that $d^{t}(u) \geq 9$ and define $t(u)$ as the number of neighbours $v$ of $u$ in $H$ such that $d^{t}(v)=7$. Lemma 19 states that $d^{t}(u) \geq 8+t(u)$. In particular, $d^{t}(u)+\sum_{v \in N_{H}(u)} d^{t}(v) \geq 8$.

Thus, let $S_{9^{+}}$(resp. $S_{8}, S_{7}$ ) be the set of non-isolated vertices with total degree at least 9 (resp. exactly 8, exactly 7 ). Let $S_{I}$ denote the set of isolated vertices of $H$. Observe that $\left\{S_{9^{+}}, S_{8}, S_{7}, S_{I}\right\}$ form a partition of $V(G)$ and then:

$$
\begin{aligned}
\sum_{v \in V(G)} d^{t}(v)= & \sum_{v \in S_{9^{+}}} d^{t}(v)+\sum_{v \in S_{8}} d^{t}(v)+\sum_{v \in S_{7}} d^{t}(v)+\sum_{v \in S_{I}} d^{t}(v) \\
& \geq 8 \cdot\left|S_{8}\right|+8 \cdot\left|S_{9^{+}} \cup S_{7}\right|+7 \cdot \operatorname{isol}(H) \\
& =8 \cdot|V(G)|-\operatorname{isol}(H)
\end{aligned}
$$

Proposition 21. Let $G$ be a connected plane graph and $H$ be a spanning forest of $G$. Then

$$
\sum_{v \in V(G)}\left(2 d^{t}(v)-14\right)+\sum_{f \in F(G)}(d(f)-6)=-12-8 \operatorname{comp}(H) .
$$

Proof. Since $H$ is a spanning forest of $G$, note that $|E(H)| \leq|V(G)|-\operatorname{comp}(H)$. Consequently, by using Euler's Formula, we have that:

$$
\begin{aligned}
\sum_{v \in V(G)}\left(2 d^{t}(v)-14\right)+\sum_{f \in F(G)}(d(f)-6) \\
\quad=2 \sum_{v \in V(G)} d_{G}(v)+4 \sum_{v \in V(G)} d_{H}(v)-14|V(G)|+2|E(G)|-6|F(G)| \\
\quad=4|E(G)|+8|E(H)|-14|V(G)|+2|E(G)|-6|F(G)| \\
\leq 6|E(G)|-6|V(G)|-6|F(G)|-8 \operatorname{comp}(H)=-12-8 \operatorname{comp}(H) .
\end{aligned}
$$

We are now ready to prove Theorem 7 .

Proof of Theorem \%. Let $(G, H)$ be a minimal counter-example with respect to the number of vertices. It is easy to check that the theorem holds when $|V(G)| \leq 3$. Hence we have $|V(G)| \geq 4$, and $(G, H)$ is $(7,2)$-minimal. By Lemma 15, $G$ must be connected.

Set $\Sigma:=\sum_{v \in V(G)}\left(2 d^{t}(v)-14\right)+\sum_{f \in F(G)}(d(f)-6)$. Proposition 21 states that $\Sigma \leq-12-8 \operatorname{comp}(H)$. We prove that such a counter-example does not exist by finding a contraction to this fact.

By Corollary 20, recall that $\sum_{v \in V(G)} d^{t}(v) \geq 8|V(G)|-\operatorname{isol}(H)$. Consequently, $\sum_{v \in V(G)}\left(2 d^{t}(v)-\right.$ 14) $\geq 2|V(G)|-2$ isol $(H)$.

Since $G$ has no cycle of length 4 or $5, \sum_{f \in F(G)}(d(f)-6) \geq-3 \tau(G) \geq-|E(G)| \geq 4-2|V(G)|$, by Lemmas 12 and 13. Combining these inequalities, we get $\Sigma \geq 4-2 \operatorname{isol}(H) \geq 4-2 \operatorname{comp}(H)$, because $\operatorname{isol}(H) \leq \operatorname{comp}(H)$ for any graph $H$.

Hence $4-2 \operatorname{comp}(H) \leq \Sigma \leq-12-8 \operatorname{comp}(H)$, which is contradiction because $\operatorname{comp}(H)$ is positive. 


\section{Path forest backbone}

Recall that a path forest is a forest whose connected components are paths. The aim of this section is to prove the following.

Theorem 8. If $G$ is a planar graph without cycles of length 4 and 5 , and $H$ is a path forest, then $C B C_{2}(G, H) \leq 6$.

In order to proof this theorem, we also consider a minimum counter-example of Theorem 8 . We also consider that $G$ is embedded in the plane, so that its face set is defined. In Subsection 4.1, we establish some properties of this counter-example. Then, in Subsection 4.2, we use these properties to derive a contradiction via the Discharging Method.

We first need some definitions. For convenience, in this section we often abbreviate circular 2backbone 6-colouring by colouring. With a slight abuse of notation, we also refer to the set of colours as $\mathbb{Z}_{6}$ so that the modulo operation is already defined (recall that initially the set of colours of a proper $k$-colouring is $\{1, \ldots, k\})$. We also write $[c]$ instead of $[c]^{6}$. Moreover, we say that two colours $a$ and $b$ are opposite if $|a-b|_{6}=3$.

Recall that $\left(G^{\prime}, H^{\prime}\right)$ is a subpair of $(G, H)$ if $H^{\prime} \subseteq H$ and $G^{\prime} \subseteq G$. We say that $\left(G^{\prime}, H^{\prime}\right)$ is an induced subpair of $(G, H)$ if $V\left(H^{\prime}\right)=V\left(G^{\prime}\right)$ and $H^{\prime}=H\left[V\left(G^{\prime}\right)\right]$ and $G^{\prime}=G\left[V\left(G^{\prime}\right)\right]$ are the corresponding induced subgraphs. A configuration of $(G, H)$ is an induced subpair in which the total degree of some of the vertices is constrained to given values.

In the remainder of this section, we represent a configuration $\left(G^{\prime}, H^{\prime}\right)$ by a representation of the graph $G^{\prime}$ with the edges of $H^{\prime}$ in bold and a number $t$ inside the circle corresponding to vertex $v$ if the total degree of $v$ must be equal to $t$, except for the last configuration where we use $\leq 8$ inside a circle to represent that the corresponding vertex has total degree at most 8 .

Let $C=\left(G^{\prime}, H^{\prime}\right)$ be a configuration of $(G, H)$. A vertex of $C$ whose degree is not constrained to some value is called external. The set of external vertices of $C$ is by $\operatorname{Ext}(C)$. The vertices of $C$ whose degree is fixed to a number are the internal vertices of $C$ and they form the set $\operatorname{Int}(C)$. Thus, $\operatorname{Ext}(C)$ and $\operatorname{Int}(C)$ form a partition of $V\left(G^{\prime}\right)$. We emphasize that, in all figures of configurations in this section, we depict all neighbours of an internal vertex.

In order to reach a contradiction and prove that no minimum counter-example to Theorem 8 exists, we show that several configurations are forbidden. By forbidden we mean that for these configurations one may extend some circular 2-backbone 6-colouring of $(G, H)-\operatorname{Int}(C)$ to $(G, H)$.

One last notion we require is the following: given a configuration $C$ of $(G, H)$ and a vertex $x \in \operatorname{Ext}(C)$, we say that two colourings $c_{1}, c_{2}$ of $(G, H)-\operatorname{Int}(C)$ are $C$-twin at $x$, if $c_{1}(x) \neq c_{2}(x)$ and $c_{1}\left(x^{\prime}\right)=c_{2}\left(x^{\prime}\right)$, for every $x^{\prime} \in \operatorname{Ext}(C) \backslash\{x\}$ (recall that by colouring we now mean circular 2-backbone 6-colouring).

\subsection{Properties of a minimal counter-example}

In the remainder of this section, $(G, H)$ is always a minimum counter-example to Theorem 8 . It means that $G$ is planar containing no cycles on 4 or 5 vertices, $H \subseteq G$ is a spanning path forest of $G$ and $(G, H)$ is a minimal (6,2)-pair. In particular, recall that for every vertex set $S \subseteq V(G),(G, H)-S$ admits a circular 2-backbone 6-colouring and $(G, H) \backslash e$ also does, for every edge $e \in E(G)$.

We now establish some properties of $(G, H)$. First recall that Lemmas 12, 16 and 17 yield the following.

Property 22. Every vertex $v \in V(G)$ is incident to at most $\lfloor d(v) / 2\rfloor$ triangles.

Property 23. For each vertex $v \in V(G), d^{t}(v) \geq 6$.

Property 24. If $u v \in E(H)$, then $d^{t}(u)+d^{t}(v) \geq 14$.

We now study more deeply the structure of $(G, H)$. Let $C_{0}$ be a configuration consisting of path $u v w$ in $H$ such that $u w \notin E(G)$ and $d^{t}(v)=6$, i.e. $\operatorname{Int}(C)=\{v\}$ and $\operatorname{Ext}(C)=\{u, w\}$. Let $C_{0}^{\prime}$ be the configuration $C_{0}$ when the edge $u w \in E(G)$ exists (see Figure 1).

Property 25. If $(G, H)$ contains a configuration $C \in\left\{C_{0}, C_{0}^{\prime}\right\}$, then

(i) there is no pair of $C$-twin colourings at $u$, and 

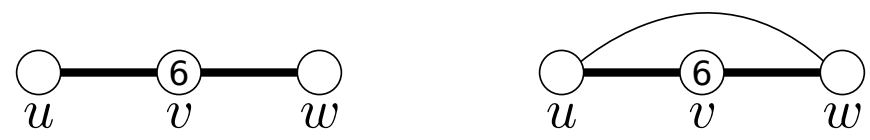

Figure 1: The configurations $C_{0}$ (left) and $C_{0}^{\prime}$ (right).

(ii) if $C=C_{0}^{\prime}$ and $u$ is a node in $H$, then $d^{t}(u) \geq 9$.

Proof. (i) Suppose, for a contradiction, that there exist two colourings $c$ and $c^{\prime}$ that are $C$-twin at $u$. One colour of $\left\{c(u), c^{\prime}(u)\right\}$, say $c(u)$, is not opposite to $c(w)=c^{\prime}(w)$. Thus $F=[c(u)] \cup[c(w)] \neq \mathbb{Z}_{6}$. Hence, choosing $c(v)$ in $\mathbb{Z}_{6} \backslash F$, we obtain a colouring of $(G, H)$, a contradiction.

(ii) Suppose again for a contradiction that $C=C_{0}^{\prime}, u$ is a node and $d^{t}(u) \leq 8$. Due to the minimality of $(G, H),\left(G^{\prime}, H^{\prime}\right)=(G, H) \backslash\{u v, u w\}$ has a circular 2-backbone 6-colouring $c$. Since $d_{\left(G^{\prime}, H^{\prime}\right)}^{t}(u) \leq 4$, vertex $u$ has at least two available colours with respect to $c$. Moreover, since $u$ is a node (i.e. a vertex of degree at least 2 in $H$ ) there are three consecutive colours in $\mathbb{Z}_{6}$ that are forbidden to $u$. So there are at least two available colours at $u$ that are not opposite. Hence one can assign to $u$ one of its available colours that does not belong to $\{c(w), c(w)+3\}$ and there would be at least one available colour to extend $c$ to $v$. Consequently there is a circular 2-backbone 6-colouring of $(G, H)$, a contradiction.

Let $C_{1}$ be a configuration on 5 vertices $u^{\prime}, u, v, v^{\prime}$ and $u^{\prime \prime}$, such that $u^{\prime} u v v^{\prime}$ form an induced path $P_{1}$ in $G$ and in $H, u^{\prime \prime}$ is not a neighbour of a vertex of $P_{1}$ in $H$, but it has exactly two neighbours of $P_{1}$ in $G: u$ and $v$. Moreover, the total degree of $u$ and $v$ must be equal to 7 , i.e. $\operatorname{Int}\left(C_{1}\right)=\{u, v\}$ (see Figure 2).

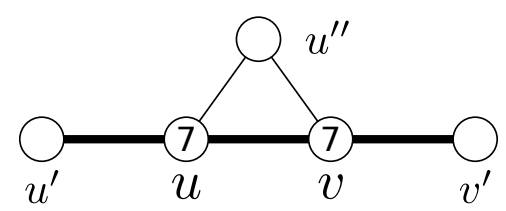

Figure 2: The configuration $C_{1}$.

Property 26. If $(G, H)$ contains a configuration $C_{1}$, then

(i) there is no pair of $C_{1}$-twin colourings at $u^{\prime}$, and

(ii) $d^{t}\left(u^{\prime}\right) \geq 8$.

Proof. (i) Suppose for a contradiction that $(G, H)-\{u, v\}$ admits two $C_{1}$-twin colourings $c$ and $c^{\prime}$ at $u^{\prime}$. One of them, say $c$, satisfies $c\left(u^{\prime}\right) \neq c\left(v^{\prime}\right)$. Note that $c\left(u^{\prime \prime}\right)$ is forbidden to $u$ and $v$. Consequently, with respect to the colouring $c$, the sets $\operatorname{Av}(u)$ and $\operatorname{Av}(v)$ of available colours at $u$ and $v$ have size at least 2 and they are not equal, since $c\left(u^{\prime}\right) \neq c\left(v^{\prime}\right)$. Thus we can choose $c(u) \in \operatorname{Av}(u)$ and $c(v) \in \operatorname{Av}(v)$ so that $|c(u)-c(v)|_{6} \geq 2$. We then obtain a colouring of $(G, H)$, a contradiction.

(ii) Suppose for a contradiction that $d_{(G, H)}^{t}\left(u^{\prime}\right) \leq 7$. The graph $(G, H)-\{u, v\}$ admits a circular 2-backbone 6-colouring $c$. The number of available colours at $u^{\prime}$ in this colouring is at least 2. Hence, we can extend $c$ into two $C_{1}$-twin colourings of $(G, H)-\{u, v\}$ at $u^{\prime}$. This contradicts (i).

Let $C_{2}$ be a configuration on eight vertices $u^{\prime}, u, v, v^{\prime}, w, u^{\prime \prime}, v^{\prime \prime}$ and $z$, such that $u^{\prime} u v v^{\prime} w$ form an induced path $P_{2}$ in $G$ and in $H$, the vertices $u^{\prime \prime}, v^{\prime \prime}$ and $z$ are not neighbours of a vertex of $P_{2}$ in $H$, but $u^{\prime \prime}$ and $v^{\prime \prime}$ have exactly two neighbours of $P_{1}$ in $G: u$ and $v, v$ and $v^{\prime}$, respectively; and the only neighbour of $z$ in $P_{2}$ is $v^{\prime}$. Moreover, $\mathrm{t} d^{t}(u)=7$ and $d^{t}(v)=d^{t}\left(v^{\prime}\right)=8$. The configuration $C_{2}^{\prime}$ is obtained from $C_{2}$ by removing $z$ and changing on the total degree of $v^{\prime}$ to 7 (see Figure 3 ). Note that $\operatorname{Int}\left(C_{2}\right)=\operatorname{Int}\left(C_{2}^{\prime}\right)=\left\{u, v, v^{\prime}\right\}$.

Property 27. If $(G, H)$ contains a configuration $C \in\left\{C_{2}, C_{2}^{\prime}\right\}$, then 

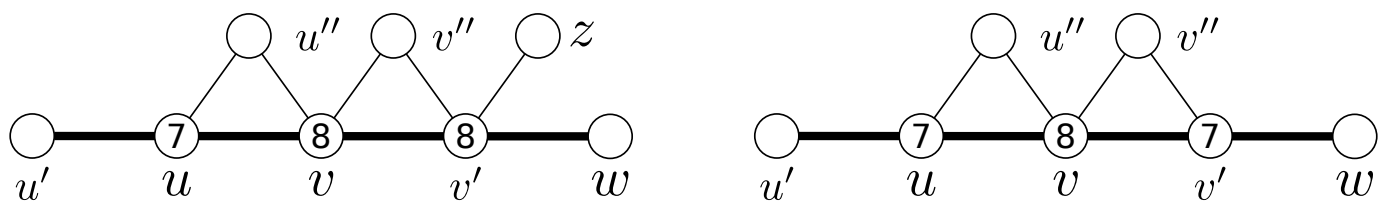

Figure 3: The configurations $C_{2}$ (left) and $C_{2}^{\prime}$ (right).

(i) there is no pair of $C$-twin colourings at $u^{\prime}$, and

(ii) $d^{t}\left(u^{\prime}\right) \geq 8$.

Proof. (i) Suppose for a contradiction that there are two $C$-twin colourings at $u^{\prime}$, say $c$ and $c^{\prime}$.

First we claim that we can extend $c$ and $c^{\prime}$ to $v^{\prime}$ with a colour $c\left(v^{\prime}\right)=c^{\prime}\left(v^{\prime}\right)$ which is not opposite to $c\left(v^{\prime \prime}\right)$. Indeed, if $c\left(v^{\prime \prime}\right) \in[c(w)]$, one we can choose $c\left(v^{\prime}\right)=c^{\prime}\left(v^{\prime}\right)$ in $\{c(w)+2, c(w)+3, c(w)+$ $4\} \backslash\left\{c(z), c\left(v^{\prime \prime}\right)+3\right\}$. Otherwise, $c\left(v^{\prime \prime}\right) \notin[c(w)]$ and in this case one can choose $c\left(v^{\prime}\right)=c^{\prime}\left(v^{\prime}\right)$ in $\{c(w)+2, c(w)+3, c(w)+4\} \backslash\left\{c\left(v^{\prime \prime}\right), c(z)\right\}$.

Consider now that $c$ and $c^{\prime}$ were extended to $v^{\prime}$ so that $c\left(v^{\prime}\right)=c^{\prime}\left(v^{\prime}\right)$ is not opposite to $c\left(v^{\prime \prime}\right)$. If $\left|c\left(v^{\prime}\right)-c\left(v^{\prime \prime}\right)\right|_{6}=1$, then $c\left(v^{\prime \prime}\right)$ is forbidden to $v$ by both $v^{\prime \prime}$ and $v^{\prime}$. So this case behaves exactly as if the edge $v v^{\prime \prime}$ did not exist in $C$ and we have a configuration equivalent $C_{1}$. Thus, similarly to the proof of Property 26-(i), one can derive that no pair of $C$-twin colourings at $u^{\prime}$ exists. Henceforth $\left|c\left(v^{\prime}\right)-c\left(v^{\prime \prime}\right)\right|_{6}=2$. Without loss of generality, assume that $c\left(v^{\prime \prime}\right)=1$ and $c\left(v^{\prime}\right)=3$. Observe that $\operatorname{Av}(v)=\{5,6\} \backslash\left\{c\left(u^{\prime \prime}\right)\right\}$ for both colourings $c$ and $c^{\prime}$.

We distinguish few cases according to the value of $c\left(u^{\prime \prime}\right)$. In each of them, we extend $c$ to a colouring of $(G, H)$, which is a contradiction.

- Case 1: $c\left(u^{\prime \prime}\right)=1$. Recall that $c$ and $c^{\prime}$ are $C$-twin colourings at $u^{\prime}$. Thus, without loss of generality, assume that $c\left(u^{\prime}\right) \neq 3$. One can then choose $c(u) \in[3] \backslash\left[c\left(u^{\prime}\right)\right]$ and $c(v) \in\{5,6\} \backslash[c(u)]$.

- Case 2: $c\left(u^{\prime \prime}\right) \in\{2,3\}$. The set $\operatorname{Av}(u)$ of available colours at $u$ is $\mathbb{Z}_{6} \backslash\left[c\left(u^{\prime}\right)\right] \cup\left\{c\left(u^{\prime \prime}\right)\right\}$, in the colouring $c$. Observe that $\operatorname{Av}(v)=\{5,6\},|\operatorname{Av}(u)| \geq 2$ and $\operatorname{Av}(u) \neq \operatorname{Av}(v)$, since $u^{\prime}$ forbids three consecutive colours and $c\left(u^{\prime \prime}\right) \in\{2,3\}$. Thus one can find $c(u) \in \operatorname{Av}(u)$ and $c(v) \in \operatorname{Av}(v)$ so that $|c(u)-c(v)|_{6} \geq 2$.

- Case 3: $c\left(u^{\prime \prime}\right) \in\{4,6\}$. Without loss of generality, we may assume that $c\left(u^{\prime}\right) \neq 2$. Then, choose $c(u) \in[2] \backslash\left[c\left(u^{\prime}\right)\right]$ and set $c(v)=5$.

- Case 4: $c\left(u^{\prime \prime}\right)=5$. Without loss of generality, assume that $c\left(u^{\prime}\right) \neq 3$. Then, one can choose $c(u) \in[3] \backslash\left[c\left(u^{\prime}\right)\right]$ and set $c(v)=6$.

(ii) Suppose for a contradiction that $d_{(G, H)}^{t}\left(u^{\prime}\right) \leq 7$. The graph $(G, H)-\left\{u, v, u^{\prime}, v^{\prime}\right\}$ admits a colouring $c$. The number of available colours at $u^{\prime}$ in $c$ is at least 2. Hence, we can extend $c$ into two $C$-twin colourings at $u^{\prime}$. This contradicts (i).

Let $C_{3}$ be a configuration of $(G, H)$ on four vertices $u, w, v$ and $z$ such that the only edges of $H$ are $u w$ and $v w$ and the edges in $E(G) \backslash E(H)$ are $u v$ and $w z$. Moreover, the total degree of $w$ must be 7 (see Figure 4).

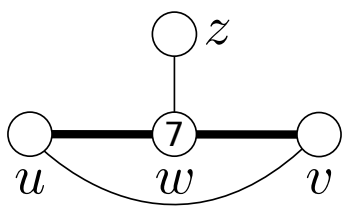

Figure 4: Configuration $C_{3}$

Property 28. If $(G, H)$ contains a configuration $C_{3}$, then 
(i) $d^{t}(u) \geq 7$, and

(ii) if $u$ is a node, then $d^{t}(u) \geq 8$.

Proof. Let $c$ be a colouring of $\left(G^{\prime}, H^{\prime}\right)=(G, H) \backslash\{u w, u v\}$. Without loss of generality, we may assume that $c(v)=1$ and $c(z) \notin\{2,3\}$.

Suppose for a contradiction that $d_{(G, H)}^{t}(u) \leq 6$ or that $u$ is a node and $d_{(G, H)}^{t}(u)=7$. If $d_{(G, H)}^{t}(u) \leq 6$, $u$ has at least four available colours in $c$, otherwise $u$ has three available colours which are consecutive. In both cases, we can assign to $u$ a colour $c(u) \in\{2,5,6\}$. If $c(u) \in\{5,6\}$, then setting $c(w)=3$, we obtain a colouring of $(G, H)$, a contradiction. If $c(u)=2$, then choosing $c(w) \in\{4,5\} \backslash\{c(z)\}$, we obtain a colouring of $(G, H)$, a contradiction.

Let $C_{4}$ be a configuration of $(G, H)$ on eight vertices $u, u^{\prime}, u^{\prime \prime}, v, v^{\prime}, v^{\prime \prime}, w$ and $z$ such that the only edges of $H$ are $u^{\prime} u, u w, w v$ and $v v^{\prime}$ and the only edges in $E(G) \backslash E(H)$ are $u^{\prime \prime} u, u v, v v^{\prime \prime}$ and $w z$. The vertices of $\operatorname{Int}\left(C_{4}\right)$ are $u, w$ and $v$ and they must have total degree equal to 8,7 and 8 , respectively (see Figure 5).

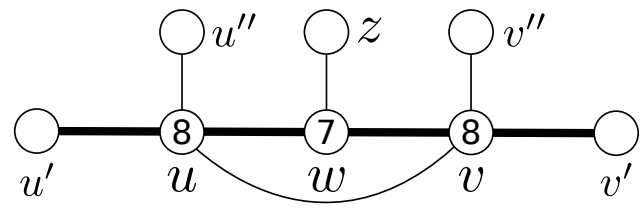

Figure 5: Configuration $C_{4}$

Property 29. If $(G, H)$ contains a configuration $C_{4}$, then

(i) $d^{t}\left(u^{\prime}\right) \geq 8$, and

(ii) if $u^{\prime} u^{\prime \prime} \in E(G-H)$ and $u^{\prime}$ is a node, then $d^{t}\left(u^{\prime}\right) \geq 9$.

Proof. Let us first prove a claim that we use to prove both statements.

We claim that there exists no colouring $c$ of $(G, H)-\{u, v, w\}$ such that $c\left(u^{\prime \prime}\right) \neq c\left(u^{\prime}\right)+3$, no matter whether $u^{\prime} u^{\prime \prime} \in E(G) \backslash E(H)$. Let us prove this claim by contradiction and let $c$ such a colouring. Observe that $|\operatorname{Av}(u)| \geq 2$ and $|\operatorname{Av}(v)| \geq 2$, and that $\operatorname{Av}(u)$ contains two consecutive colours, since $c\left(u^{\prime}\right) \neq c\left(u^{\prime \prime}\right)+3$. Without loss of generality, we assume that $\{1,2\} \subseteq A(u)$ and that $c(z) \in\{2,3,4\}$. Since $|\operatorname{Av}(v)| \geq 2$, we first extend the colouring $c$ to $v$ by colouring $v$ with a colour $c(v) \neq 5$. Then, in order to reach a contradiction, we show that for every possible colour $c(v) \neq 5$ one can extend $c$ into a colouring of $(G, H)$ :

- Case 1: $c(v) \in\{1,2,3\}$. Set $c(w)=5$ and choose $c(u) \in\{1,2\} \backslash\{c(v)\}$.

- Case 2: $c(v)=4$. Set $c(u)=2$ and $c(w)=6$.

- Case 3: $c(v)=6$. Set $c(u)=1$ and choose $c(w) \in\{3,4\} \backslash\{c(z)\}$.

This completes the proof of the claim.

(i) By contradiction, suppose that $d^{t}\left(u^{\prime}\right) \leq 7$. Let $c$ be a colouring of $(G, H)-\{u, v, w\}$. Since $u$ is not coloured by $c$ and $u^{\prime} u \in E(H)$, vertex $u^{\prime}$ has at least two available colours in $c$. Thus one can, if necessary, recolour $u^{\prime}$ so that $c\left(u^{\prime \prime}\right) \neq c\left(u^{\prime}\right)+3$, contradicting the previous claim.

(ii) By contradiction, suppose that $u^{\prime} u^{\prime \prime} \in E(G-H)$ and $u^{\prime}$ is a node, and $d^{t}\left(u^{\prime}\right) \leq 8$. Let $c$ be a colouring of $(G, H)-\{u, v, w\}$ and $t^{\prime}$ be the neighbour of $u^{\prime}$ in $H$ that is distinct from $u$. By definition, we have that $c\left(t^{\prime}\right) \notin\left[c\left(u^{\prime}\right)\right]$ and by the previous claim, $c\left(u^{\prime \prime}\right)=c\left(u^{\prime}\right)+3$. Combining these facts, one deduces that $c\left(u^{\prime \prime}\right) \in\left[c\left(t^{\prime}\right)\right]$. Moreover, since $d^{t}\left(u^{\prime}\right) \leq 8, u^{\prime}$ has at most one neighbour in $G-\left\{u, u^{\prime \prime}, t^{\prime}\right\}$ that is already coloured by $c$. Therefore, since $u$ is not yet coloured, $u^{\prime}$ has at least two available colours in $c$. Hence, one can recolour $u^{\prime}$ with a colour distinct from $c\left(u^{\prime \prime}\right)+3$, contradicting to the above claim. 


\subsection{Proof of Theorem 8}

The proof uses the Discharging Method. As again consider in this section that $(G, H)$ is a minimal counter-example to Theorem 8 and we also consider that $G$ is embedded in the plane, so that we can refer to its faces. We first assign a initial weight each vertex and face of $G$, and prove that the total initial weight is negative. We then apply some discharging rules that do not change the total weight. Finally, using the properties established in the previous section, we prove that the final weight of each vertex and face of $G$ is non-negative, which contradicts the negativity of the total weight. This implies that no such a counter-example exists and proves Theorem 8.

\subsubsection{Initial weight}

Let us define a function initial weights $\varphi: V(G) \cup F(G) \rightarrow \mathbb{Z}$ of the vertices and faces of $G$ as follows:

- If $v$ is an isolated vertex in $H$, i.e. $d_{H}(v)=0$, then $\varphi(v)=2 d^{t}(v)-6$;

- If $v$ is a leaf in $H$, i.e. $d_{H}(v)=1$, then $\varphi(v)=2 d^{t}(v)-10$;

- If $v$ is a node in $H$, i.e. $d_{H}(v)=2$, then $\varphi(v)=2 d^{t}(v)-14$;

- Every face $f \in F(G)$ has weight $\varphi(f)=d(f)-6$.

Observe that the only faces with negative weight are the 3 -faces for which the weight is -3 , since $G$ has no $C_{4}$ nor $C_{5}$. Let $\Phi=\sum_{v \in V} \varphi(v)+\sum_{f \in F} \varphi(f)$ be the total initial weight. The following lemma shows that $\Phi$ is negative.

Lemma 30. The total initial weight $\Phi$ equals -12 .

Proof. We have

$$
\Phi=\sum_{v \in V}\left(2 d^{t}(v)-14\right)+\sum_{f \in F}(d(f)-6)+4 \operatorname{leaf}(H)+8 \operatorname{isol}(H) .
$$

We shall use Euler's Formula, and the three following easy facts, whose proof is left to the reader.

$$
\begin{gathered}
\sum_{v \in V} d(v)=\sum_{f \in F} d(f)=2|E(G)| \\
\sum_{v \in V} d^{t}(v)=2|E(G)|+4|E(H)| \\
|E(H)|=|V(G)|-\operatorname{leaf}(H) / 2-\operatorname{isol}(H)
\end{gathered}
$$

By (4) and (5), we have

$$
\Phi=6|E(G)|+8|E(H)|-14|V(G)|-6|F(G)|+4 \operatorname{leaf}(H)+8 \operatorname{isol}(H)
$$

Then using (6), we get $\Phi=6|E(G)|-6|F(G)|-6|V(G)|$. Finally, by Euler's Formula, we obtain $\Phi=-12$.

\subsubsection{Discharging rules}

We now apply a set of discharging rules that we apply to convert $\varphi$ into a final function of weights $\varphi^{\prime}$ in such a way that no charge is lost, i.e. the total charge should be the same in $\varphi^{\prime}$.

- Rule 1: Each vertex sends one unit of charge to each of its incident 3-faces.

- Rule 2: Each $q$-face, for every $q \geq 6$, sharing two consecutive backbone edges with a 3-face sends one unit of charge to the node of total degree 6 this 3 -face, if it exists.

- Rules 3: For each of the configurations depicted in Figure 6, vertex $s$ sends one unit of charge to vertex $t$, if $\varphi(s) \geq 4$. 


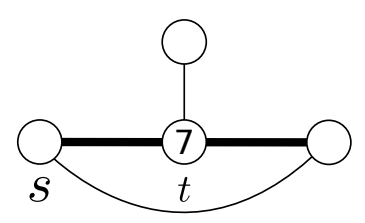

(a) Rule 3.1

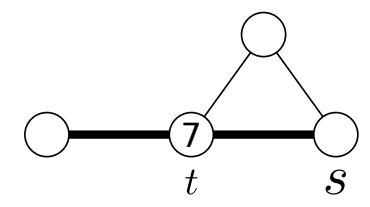

(c) Rule 3.3

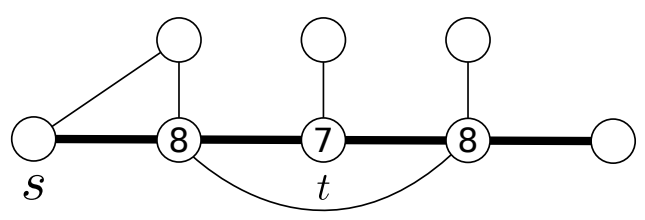

(b) Rule 3.2

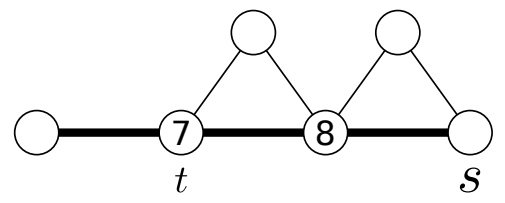

(d) Rule 3.4

Figure 6: Rules 3. To send charge $s$ must have initial weight at least 4 .

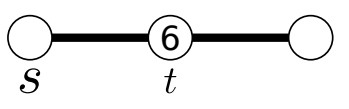

(a) Rule 4.1

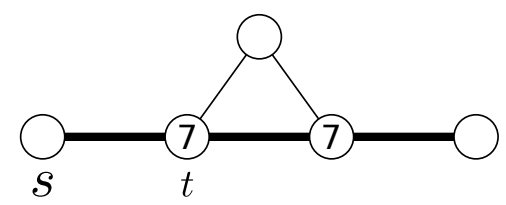

(c) Rule 4.3

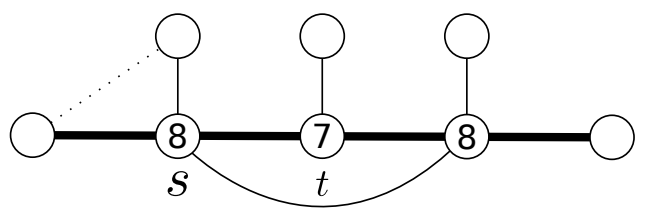

(b) Rule 4.2

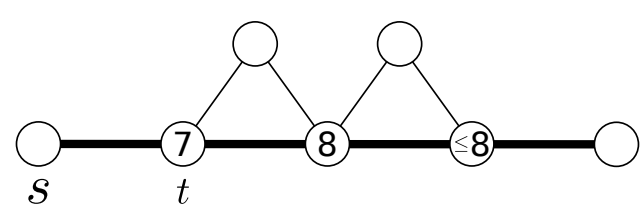

(e) Rule 4.5

Figure 7: Rules 4. The dotted line means that the edge belongs to $E(G)$.

- Rules 4: For each of the configurations depicted in Figure 7, vertex $s$ sends one unit of charge to vertex $t$.

Lemma 31. Whenever one of the Rules 4 is applied, the vertex s has total degree at least 8.

Proof. The total degree of $s$ is imposed to be 8 in Rules 4.2 and 4.4. It is at least 8 in Rules 4.1, 4.3, and 4.5 by Properties 24, 26, and 27, respectively.

Lemma 32. By Rules 3 and 4, a node sends at most two units of charge and a leaf sends at most one unit of charge.

Proof. In Rules 3 and 4, a vertex $s$ sends charge to a vertex a distance at most 2 in $H$. Moreover, each time its sends to a vertex $t$ at distance 2 in $H$, then the common neighbour of $s$ and $t$ in $H$ does not send nor receive any charge by Rules 3 or 4 . Therefore, by Rules 3 and 4, a vertex sends at most 1 in each direction along its path in $H$. 


\subsubsection{Final weight}

We now show that all vertices and all faces have a non-negative final weight. Recall that we denote by $\varphi^{\prime}$ the final weight function after all applications of the previously described rules.

Lemma 33. Every face $f \in F(G)$ has a non-negative final weight.

Proof. If $f$ is a 3 -face, then $\varphi(f)=-3$, and $f$ receives 1 for each of its vertices by Rule 1 . So $\varphi^{\prime}(f)=0$.

If $f$ is not a 3 -face, then $f$ has degree at least 6 . So we have $\varphi(f) \geq 0$. However, we have to be sure that Rule 2 , the only rule to change the weight of faces, cannot create a face with negative weight. Assume that $f$ shares two backbone edges with $k 3$-faces $T_{i}, 1 \leq i \leq k$. Recall that no two triangles share an edge, by Lemma 12. Thus, one can find a cycle $C$ in $G$ by replacing the two backbone edges in $T_{i} \cap f$ (and their common endvertex) by the third edge of $T_{i}$. The cycles obtained from $f$ by taking $k$ or $k-1$ of such shortcuts are cycles of length $d(f)-k$ and $d(f)-k+1$, respectively. Since $G$ has no cycles of length 4 and 5 , it follows that $d(f)-k \geq 6$. Hence $\varphi^{\prime}(f)=d(f)-6-k \geq 0$.

Lemma 34. If $x$ is an isolated vertex, then $\varphi^{\prime}(x) \geq 0$.

Proof. Observe that the charge of $x$ is $\varphi(x)=2 d^{t}(x)-6 \geq d^{t}(x)$, by Property 23. No isolated vertex is involved in Rules 2, 3 or 4, so $x$ is concerned only by Rule 1 . Since $x$ is adjacent to at most $\lfloor d(x) / 2\rfloor$ 3 -faces, by Property 22, we deduce that $\varphi^{\prime}(x) \geq d^{t}(x)-\lfloor d(x) / 2\rfloor \geq 0$.

Lemma 35. If $x$ is a leaf, then $\varphi^{\prime}(x) \geq 0$.

Proof. Recall that $\varphi(x)=2 d^{t}(x)-10$. Vertex $x$ sends at most one unit of charge by Rules 3 and 4 , by Lemma 32. By Property 22, $x$ is incident to at most $\lfloor d(x) / 2\rfloor 3$-faces. Since $\lfloor d(x) / 2\rfloor=\left\lfloor d^{t}(x) / 2\right\rfloor-1$, we have $\varphi^{\prime}(x) \geq 2 d^{t}(x)-10-\left(\left\lfloor d^{t}(x) / 2\right\rfloor-1\right)-1$. Thus, if $d^{t}(x) \geq 7$ then $\varphi^{\prime}(x) \geq 0$.

Suppose now that $d^{t}(x)<7$, so $d^{t}(x)=6$ by Property 23 . We have $\varphi(x)=2$, so $x$ does not send charge by Rules 3. By Lemma 31, $x$ does not send charge by Rules 4 . Moreover $x$ is incident to at most two 3 -faces. So we have $\varphi^{\prime}(x) \geq \varphi(x)-2 \geq 0$.

For nodes, we distinguish several cases according to their total degree.

Lemma 36. Let $x$ be a node. If $d^{t}(x) \geq 9$, then $\varphi^{\prime}(x) \geq 0$.

Proof. We have $\varphi(x)=2 d^{t}(x)-14 \geq d^{t}(x)-5$. Now $x$ gives at most 2 by Rules 3 and 4 by Lemma 32 , and 1 to every 3 -face. As $x$ is incident to at most $\lfloor d(x) / 2\rfloor=\left\lfloor d^{t}(x) / 2\right\rfloor-23$-faces (by Property 22), we have $\varphi^{\prime}(x) \geq d^{t}(x)-5-\left(\left\lfloor d^{t}(x) / 2\right\rfloor-2\right)-2 \geq\left\lceil d^{t}(x) / 2\right\rceil-5 \geq 0$.

Lemma 37. Let $x$ be a node. If $d^{t}(x)=6$, then $\varphi^{\prime}(x) \geq 0$.

Proof. $x$ sends no charge by Rules 3 because $\varphi(x)=-2$, nor by Rules 4 by Lemma 31. Moreover, by Rule 4.1, $x$ receives 2 ( 1 of each of its neighbours). Now $x$ is adjacent to at most one 3 -face by Property 22, because $d(x)=2$. If $x$ is incident to no 3-face, then it does not gives anything by Rule 1 . So $\varphi^{\prime}(x)=\varphi(x)+2=0$. If $x$ is incident to a 3 -face, then it gives 1 to this 3 -face by Rule 1 , but it also receives 1 by Rule 2 . Hence $\varphi^{\prime}(x)=\varphi(x)+2-1+1=0$.

Lemma 38. Let $x$ be a node. If $d^{t}(x)=7$, then $\varphi^{\prime}(x) \geq 0$.

Proof. $x$ sends no charge by Rules 3 because $\varphi(x)=0$, nor by Rules 4 by Lemma 31. Moreover, $x$ is adjacent to at most one 3 -face by Property 22 , because $d(x)=3$.

If $x$ is incident to no 3 -face, then it does not send any charge, so $\varphi^{\prime}(v) \geq \varphi(x)=0$.

Assume now that $x$ is incident to a 3 -face $T$. Therefore $x$ sends a charge of 1 by Rule 1 .

- If $T$ contains the two backbone edges incident to $x$, then we are in a Configuration $C_{3}$. Let us use the notation of the Figure 4 , so our vertex $x$ is $w$. Observe that $d^{t}(u) \geq 7$ and $d^{t}(v) \geq 7$ by Property 24.

- If $\varphi(u) \geq 4$ or $\varphi(v) \geq 4$, then by Rule $3.1, x$ receives at least 1 . Hence $\varphi^{\prime}(x) \geq \varphi(x)-1+1=0$ 
- If $\varphi(u)<4$ and $\varphi(v)<4$, then $u$ and $v$ are nodes. Hence $d^{t}(u) \leq 8$ and $d^{t}(v) \leq 8$. Consequently, by Property $28, d^{t}(u)=d^{t}(v)=8$. Thus, we are in a Configuration $C_{4}$ with $x=w$. Let us use the notation of Figure 5. If $u^{\prime}$ is not adjacent to $u^{\prime \prime}$, then $x$ receives 1 from $u$ by Rule 4.2. If $u^{\prime} u^{\prime \prime}$ is an edge, then $u^{\prime}$ is either a leaf with total degree at least 7 (by Property 28), or a node with total degree at least 9 (by Property 29). In both cases, $\varphi\left(u^{\prime}\right) \geq 4$, so $x$ receives 1 from $u^{\prime}$ by Rule 3.2. So $\varphi^{\prime}(x) \geq \varphi(x)-1+1 \geq 0$.

- If $T$ contains only one of the backbone edges incident to $x$, then we are in Configuration $D$ depicted Figure 8. Let us use the notation of this figure. By Property 24, both $u^{\prime}$ and $v$ have total degree at least 7 .

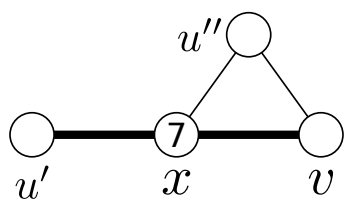

Figure 8: Configuration $D$

- If $\varphi(v) \geq 4$, then $x$ receives 1 from $v$ by Rule 3.3. Hence $\varphi^{\prime}(x) \geq \varphi(x)-1+1=0$.

- If $\varphi(v)=2$, then $v$ is a node, so $d^{t}(v)=8$. Let $v^{\prime}$ be the neighbour of $v$ in $H$ that is distinct from $x$.

* If $T$ is the sole 3 -face to which $v$ is incident, then $x$ receives 1 from $v$ by Rule 4.4. Hence $\varphi^{\prime}(x) \geq \varphi(x)-1+1=0$.

* If $v$ is incident to a 3 -face distinct from $T$, then this 3 -face contains $v^{\prime}$. If $\varphi\left(v^{\prime}\right) \geq 4$, then $x$ receives 1 from $v^{\prime}$ by Rule 3.4. Otherwise $v$ is a node and $d^{t}\left(v^{\prime}\right) \leq 8$, so we are in Configuration $C_{2}$ or $C_{2}^{\prime}$ with $x=u$, and $x$ receives 1 from $u^{\prime}$ by Rule $4 . \overline{5}$. In both cases, $\varphi^{\prime}(x) \geq \varphi(x)-1+1=0$.

- If $\varphi(v)=0$, the $v$ is a node and $d^{t}(v)=7$. So we are in Configuration $C_{1}$ with $x=u$. Thus $x$ receives 1 from $u^{\prime}$ by Rule 4.5. Hence $\varphi^{\prime}(x) \geq \varphi(x)-1+1=0$.

Lemma 39. Let $x$ be a node. If $d^{t}(x)=8$, then $\varphi^{\prime}(x) \geq 0$.

Proof. We have $\varphi(x)=2$, so it does not send anything by Rule 3. Vertex $x$ sends at most 2 by Rules 4 . By Property 22, $x$ is adjacent to at most two 3 -faces, because $d(x)=4$.

If $x$ is incident to no 3 -faces, it sends a charge of at most 2 in total so $\varphi^{\prime}(x) \geq \varphi(x)-2 \geq 0$.

If $x$ is incident to two 3 -faces, then none of the Rules 4 applies. So $x$ sends a charge of at most 2 , and $\varphi^{\prime}(x) \geq \varphi(x)-2 \geq 0$.

Assume now that $x$ is incident to exactly one 3-face. If $x$ sends a charge of 0 or 1 by Rules 4 , then $\varphi^{\prime}(x) \geq \varphi(x)-1-1 \geq 0$. To complete the proof we shall now prove that $x$ cannot send a charge of 2 by Rules 4.

Suppose by contradiction that it does. Then $x$ is the vertex $s$ for two configurations $C$ and $C^{\prime}$ isomorphic to some depicted Figure 7. Observe that $C$ and $C^{\prime}$ cannot be both isomorphic to the configurations depicted in Figures $7(\mathrm{~b})$ or $7(\mathrm{~d})$, because $x$ is incident to one 3 -face. Hence one of these two configurations, say $C$, is isomorphic to either $C_{0}, C_{1}, C_{2}$, or $C_{2}^{\prime}$, depicted in Figures $7(\mathrm{a}), 7(\mathrm{c})$ and $7(\mathrm{e})$. Let $t^{\prime}$ be the vertex of $C^{\prime}$ corresponding to $t$ in Figure 7. Observe that $t^{\prime}$ is not an external vertex of $C$, because $G$ has no cycle of length 4 or 5 . Consider a colouring $c$ of $\left(G^{\prime}, H^{\prime}\right)=(G, H) \backslash\left(\operatorname{Int}(C) \cup\left\{x, t^{\prime}\right\}\right)$. We have $d_{\left(G^{\prime}, H^{\prime}\right)}^{t}\left(t^{\prime}\right) \leq d_{(G, H)}^{t}\left(t^{\prime}\right)-3 \leq 4$. Hence the set $A\left(t^{\prime}\right)$ of available colours at $t^{\prime}$ has size at least 2. Furthermore, $d_{\left(G^{\prime}, H^{\prime}\right)}^{t}(x) \leq d_{(G, H)}^{t}(x)-6=2$, so the set $A(x)$ of available colours at $x$ has size at least 4 . Hence there exist two distinct colours $c_{1}$ and $c_{2}$ of $A(x)$ and two colours $c_{1}^{\prime}$ and $c_{2}^{\prime}$ in $A\left(t^{\prime}\right)$ (not necessarily distinct) such that $c_{1} \notin\left[c_{1}^{\prime}\right]$ and $c_{2} \notin\left[c_{2}^{\prime}\right]$. Thus there exists two $C$-twin colourings at $x$. This contradicts Properties 25-(i), 26-(i), or 27-(i).

Lemmas 33, 34, 35,36, 37, 38, and 39 show that all faces and vertices have non-negative final weight. Thus $\Phi \geq 0$. This contradicts Lemma 30 and completes the proof of the theorem. 


\section{References}

[1] Appel, K., AND HAKen, W. Every planar map is four colorable. Bulletin of Americain mathematical society $82(1976)$.

[2] Bondy, J. A., And Murty, U. S. R. Graph theory, vol. 244 of Graduate Texts in Mathematics. Springer, New York, 2008.

[3] Broersma, H., Fomin, F. V., Golovach, P. A., and Woeginger, G. J. Backbone colorings for graphs: Tree and path backbones. Journal of Graph Theory 55, 2 (June 2007), 137-152.

[4] Campos, V., Havet, F., Sampaio, R., and Silva, A. Backbone colouring: Tree backbones with small diameter in planar graphs. Theoretical Computer Science 487, 0 (2013), 50 - 64.

[5] Grötzsch, H. Zur Theorie der diskreten Gebilde. VII. Ein Dreifarbensatz für dreikreisfreie Netze auf der Kugel. Wiss. Z. Martin-Luther-Univ. Halle-Wittenberg. Math.-Nat. Reihe 8 (1958/1959), $109-120$.

[6] Havet, F., King, A. D., Liedloff, M., And Todinca, I. (Circular) backbone colouring: Forest backbones in planar graphs. Discrete Applied Mathematics 169 (2014), 119-134.

[7] Steinberg, R. The state of the three color problem. In Quo vadis, graph theory?, vol. 55 of Ann. Discrete Math. North-Holland, Amsterdam, 1993, pp. 211-248. 